Yi-Jui Liu, PhD

Cheng-Yu Chen, MD Hsiao-Wen Chung, PhD

Ing-Jye Huang, BS Chang-Shin Lee, PhD Shy-Chyi Chin, MD Michelle Liou, PhD

\section{Index terms:}

Brain, infarction, 13.4352, 13.781

Brain, MR, 13.121413, 13.121414,

$13.121416,13.12143,13.12144$

13.12145

Magnetic resonance (MR), perfusion study, 13.12144

Published online before print

10.1148/radiol.2292020639

Radiology 2003; 229:366-374

Abbreviations:

$A D C=$ apparent diffusion coefficient ANOVA $=$ analysis of variance

$\mathrm{CBV}=$ cerebral blood volume

$\mathrm{NAA}=\mathrm{N}$-acetylaspartate

$\mathrm{rCBV}=$ relative $\mathrm{CBV}$

$\mathrm{TI}=$ territorial infarction

$\mathrm{WI}=$ watershed infarction

${ }^{1}$ From the Department of Electrical Engineering, National Taiwan University, Taipei, R.O.C. (Y.J.L., H.W.C., I.J.H.); Department of Radiology, Tri-Service General Hospital and National Defense Medical Center, Number 325, Section 2, ChengKung Rd, Neihu 114, Taipei, Taiwan, R.O.C. (Y.J.L., C.Y.C., H.W.C., C.S.L., S.C.C.); and Institute of Statistics Science, Academia Sinica, Taipei, Taiwan, R.O.C. (M.L.). Received May 31, 2002; revision requested July 29; final revision received April 11, 2003; accepted May 14. Supported in part by National Science Council grants NSC-89-2320-B-016-056M08 and NSC-90-2213-E-002-003. Address correspondence to C.Y.C. (email: sandy0928@seed.net.tw).

\section{Author contributions:}

Guarantor of integrity of entire study, C.Y.C.; study concepts, C.Y.C., H.W.C., C.S.L.; study design, C.Y.C., H.W.C.; literature research, Y.J.L., I.J.H.; clinical studies, C.Y.C., S.C.C., Y.J.L., H.W.C., C.S.L.; data acquisition, Y.J.L., H.W.C.; data analysis/interpretation, Y.J.L., H.W.C., C.Y.C., C.S.L.; statistical analysis, M.L.; manuscript preparation, Y.J.L., H.W.C., C.S.L.; manuscript definition of intellectual content and editing, H.W.C., C.Y.C.; manuscript revision/review, H.W.C., C.Y.C., C.S.L.; manuscript final version approval, C.Y.C.

○ RSNA, 2003
Neuronal Damage after Ischemic Injury in the Middle Cerebral Arterial Territory: Deep Watershed versus Territorial Infarction at MR Perfusion and Spectroscopic Imaging

PURPOSE: To determine the temporal patterns of neuronal injury between infarction subtypes and their possible association with changes in cerebral blood volume (CBV).

MATERIALS AND METHODS: Twenty-five patients with ischemic injuries of middle cerebral arterial territories and receiving only conservative treatments were classified into territorial infarction (TI) $(n=16)$ and watershed infarction (WI) $(n=$ 9) groups and were prospectively evaluated with longitudinal magnetic resonance (MR) examinations. Each patient underwent as many as five MR studies at various stroke stages following stroke symptom onset. Dynamic susceptibility-weighted contrast material-enhanced MR imaging was performed to yield the relative CBV (rCBV). Chemical shift imaging was used to measure the relative levels of $N$ acetylaspartate (NAA) and lactate of the ischemic brain tissue. Repeated-measures analysis of variance was used to examine the statistical significance in evolutional differences between $\mathrm{TI}$ and WI.

RESULTS: For patients with TI, rCBV followed a progressively increasing pattern, from initial low values $(0.46 \pm 0.28[S D])$ to peak high values $(1.23 \pm 0.34)$ at early chronic stage. Relative NAA level decreased to $0.40 \pm 0.24$ during acute stroke and was lost completely 4 days after ictus. Patients with WI showed consistently high rCBV throughout all stages, with residual relative NAA level $(0.53 \pm 0.25)$ even at 1 month after symptom onset. Relative lactate level of patients with $\mathrm{TI}$ was significantly higher than that of patients with $\mathrm{Wl}$ at the acute stage $(P<.01)$. Differences in the temporal changes of both rCBV and brain metabolites between $\mathrm{Tl}$ and $\mathrm{WI}$ were significant $(P<.01)$.

CONCLUSION: The different temporal patterns for stroke progression in $\mathrm{TI}$ and $\mathrm{WI}$ are associated with different evolutions of hemodynamics and neuronal injury.

(c) RSNA, 2003

Deep watershed infarction (WI) typically occurs in the supraganglionic periventricular white matter within the border zones of a single territory of one major artery (1-3). In contrast to patients with territorial infarction (TI), patients with WI often have a history of transient ischemic attack $(1,4)$, generally have better outcomes $(4,5)$, and, most important, do not benefit from acute stroke interventions such as intravenous administration of recombinant tissue-type plasminogen activator (6). In addition, findings of a recent longitudinal magnetic resonance (MR) imaging study show that the temporal evolution of the apparent diffusion coefficient (ADC) reveals distinct patterns between patients with TI 
and those with WI, which suggests that the speed of infarction progression may be strongly dependent on infarction subtypes (7). The fact that patients with TI and WI exhibit inherently different hemodynamic natures $(1,2,8)$ may be one of the important reasons for the subtype dependency. It would therefore be interesting to know whether the progress of neuronal injury, similar to ADC evolution, is dependent on infarction subtypes and whether the dependency, if present, may be associated with the difference in the underlying hemodynamics, as was found in animal models of cerebral ischemia $(9,10)$.

As a continuation of a previous investigation about ADC evolution in human subjects, this study aims at testing the hypothesis that the difference between the evolution of WI and that of TI is related to different time courses of hemodynamic changes and neuronal injury. Thus, the purpose of our study was to determine the temporal patterns of neuronal injury between infarction subtypes and to examine their possible association with changes in the cerebral blood volume (CBV).

\section{MATERIALS AND METHODS}

\section{Subjects}

A total of 25 patients (16 men and nine women; mean age, 60 years \pm 17 [SD]) with ischemic stroke in the territory of the middle cerebral artery were included in this study during a 2 -year period. The average age of the male patients was slightly higher than that of the female patients, but the difference was not statistically significant $(P=.23)$. Patients were enrolled according to the following criteria: definite clinical diagnosis of stroke, determinable timing of stroke symptom onset with history, persistent neurologic deficits, and unilateral infarction. Patients who had negative MR results, symptoms of recurrent stroke, ischemic stroke in the territory other than the middle cerebral artery, or showed evidence of acute cerebral hemorrhage were excluded from the study. Patients who received thrombolytic therapy or medication with neuroprotective agents were also excluded. All patients who met the criteria during the 2-year period were recruited. Thus, all 25 patients received only conservative treatments, that is, the time course of neuronal injury reported in this study represents the natural evolution of cerebral ischemia. The Tri-Service General Hospital review board re- viewed our study and concluded that its approval was not required. Informed consent was obtained from all patients or from the closest family members if the patient was unable to provide consent because of neurologic deficits.

To observe the evolution of cerebral ischemic infarction, as many as five MR measurements were performed for each patient at the following six stages of stroke: hyperacute (6 or fewer hours after symptom onset), acute (more than 6 hours and 2 or fewer days after symptom onset), early subacute (3-4 days after symptom onset), late subacute (7-9 days after symptom onset), early chronic (10-15 days after symptom onset), and late chronic (30-31 days after symptom onset). There were a total of $92 \mathrm{MR}$ examinations.

Classification of infarction subtypes was first determined by an experienced radiologist (C.Y.C.) from diffusionweighted images by using existing atlas templates $(3,11)$. TI was defined as the presence of an ischemic lesion that involved both the cortical and subcortical areas and was restricted to the unilateral middle cerebral arterial territory. WI was defined as the presence of an ischemic lesion in the subcortical white matter within the border zones between the superficial branches and the deep perforators of the same middle cerebral artery (7). The subtype classification of the patients was further confirmed with MR angiographic findings at their first MR examinations.

\section{MR Imaging Protocols}

All MR images were acquired at $1.5 \mathrm{~T}$ (Magnetom Vision Plus system; Siemens, Erlangen, Germany). In addition to diffusion-weighted echo-planar imaging as a routine stroke screening protocol $(4,700 / 120$ [repetition time msec/echo time msec], $256 \times 256$ matrix, 230-mm field of view, $5-\mathrm{mm}$ section thickness, 1.5-mm gap between sections, 20 sections in one acquisition, and diffusionweighted factors $b$ of 0 and $1,000 \mathrm{sec} /$ $\mathrm{mm}^{2}$ at three orthogonal gradient axes), each patient underwent dynamic susceptibility-weighted contrast material-enhanced perfusion-weighted imaging at all examination periods. For this purpose, after a manual bolus injection of $0.2 \mathrm{mmol}$ per kilogram of body weight gadopentetate dimeglumine (Magnevist; Schering AG, Berlin, Germany) at about 4-5 mL/sec through a 20 -gauge venous catheter inserted into the antecubital vein, echo-planar imaging was per- formed to acquire a series of perfusionweighted images (44-msec echo time, $230-\mathrm{mm}$ field of view, $128 \times 128$ matrix, $5-\mathrm{mm}$ section thickness, three to six sections to cover the ischemic regions, 60-75 frames per section at 1-second interval). Images were transferred to a personal computer for pixel-by-pixel analysis of the CBV. T2-weighted images with cerebrospinal fluid suppression were also obtained by using a fluid-attenuated inversion-recovery technique $(9,000 / 110$; inversion time, $2,500 \mathrm{msec}$ ) to assess the final infarct size.

Information about the temporal evolution of neuronal injury was obtained by using hydrogen 1 chemical shift imaging performed at predefined intervals, as previously described. A point-resolved spectroscopic technique was used $(1,500 / 270$, $16-\mathrm{cm}$ field of view, $16 \times 16$ phase encodings, $10-\mathrm{mm}$ section thickness, $1-\mathrm{kHz}$ spectral width). The acquisition time for the chemical shift imaging sequence was about 13 minutes, with two signals acquired. Shimming was performed before imaging to ensure field homogeneity with full width at half maximum of the spectral peaks below $10 \mathrm{~Hz}$. A volume of interest encompassing both lesion and normal areas was selected first by using three orthogonal localization images and was properly positioned to match closely the diffusion-weighted image that covered the greatest extent of the lesion. This position was confirmed by one of the radiologists (C.Y.C. or S.C.C.) before execution. Depending on lesion size, the volume of interest contained about $8 \times 8$ voxels, each of which was about $10 \times$ $10 \times 10 \mathrm{~mm}^{3}$ in volume and zero filled to $5 \times 5 \times 10 \mathrm{~mm}^{3}$ for subsequent spectral analysis. The MR protocols for this ischemic stroke study were approved by the hospital review board.

\section{Data Processing}

The relative CBV (rCBV) maps were derived from the dynamically acquired perfusion-weighted images with analysis software (Matlab; MathWorks, Natick, Mass). The signal-time curve of each pixel was first smoothed with a third-order lowpass Butterworth filter at a cutoff frequency of $0.15 \mathrm{~Hz}$ to reduce interframe noise interference. The concentration of the contrast agent was then computed by taking the negative logarithm of the time signal after subtraction of the precontrast baseline intensity (ie, concentration proportional to changes in the apparent transverse relaxation rate $1 / \mathrm{T} 2 *)(12)$. Thus, one concentration-time curve was obtained for each 


\begin{tabular}{|c|c|c|c|c|c|c|c|}
\hline $\begin{array}{l}\text { Patient } \\
\text { No. }^{*}\end{array}$ & Hyperacute & Acute & $\begin{array}{c}\text { Early } \\
\text { Subacute }\end{array}$ & $\begin{array}{c}\text { Late } \\
\text { Subacute }\end{array}$ & $\begin{array}{c}\text { Early } \\
\text { Chronic }\end{array}$ & $\begin{array}{c}\text { Late } \\
\text { Chronic }\end{array}$ & $\begin{array}{c}\text { Final Infarct } \\
\text { Volume } \\
\left(\mathrm{cm}^{3}\right)\end{array}$ \\
\hline 1 & NA & 0.480 & NA & 1.213 & 1.010 & 0.809 & 64.4 \\
\hline 2 & NA & NA & 0.672 & 0.762 & 1.377 & 1.163 & 45.2 \\
\hline 3 & NA & 0.277 & NA & 0.356 & 0.819 & 0.673 & 193.7 \\
\hline 4 & NA & NA & 0.489 & NA & 1.098 & 0.826 & 25.2 \\
\hline 5 & 0.534 & NA & 0.683 & 1.082 & 1.222 & 0.824 & 59.2 \\
\hline 6 & 0.105 & NA & 0.070 & NA & NA & 0.556 & 310.2 \\
\hline 7 & 0.411 & NA & 0.157 & 0.451 & NA & 0.373 & 327.1 \\
\hline 8 & NA & NA & 0.830 & 1.573 & 1.772 & 1.165 & 17.2 \\
\hline 9 & NA & NA & 0.880 & 1.301 & 1.492 & 0.580 & 11.1 \\
\hline 10 & NA & NA & 0.509 & 0.650 & 1.702 & 1.175 & 42.8 \\
\hline 11 & NA & 0.427 & NA & 0.518 & NA & NA & 10.5 \\
\hline 12 & NA & NA & 0.648 & 0.524 & 1.499 & NA & 8.4 \\
\hline 13 & 0.770 & NA & NA & 1.290 & 0.875 & NA & 166.2 \\
\hline 14 & NA & NA & 1.444 & NA & 1.116 & 0.966 & 48.7 \\
\hline 15 & NA & NA & 1.612 & 1.573 & 1.363 & 1.040 & 41.3 \\
\hline 16 & NA & 1.380 & 1.350 & 1.227 & NA & 0.785 & 25.8 \\
\hline 17 & NA & NA & 0.675 & 1.157 & 0.750 & 0.750 & 4.6 \\
\hline 18 & NA & 0.890 & NA & 1.130 & 1.090 & NA & 1.6 \\
\hline 19 & NA & NA & 0.987 & NA & 1.800 & 1.520 & 1.7 \\
\hline 20 & NA & NA & 1.009 & 0.952 & 1.198 & 1.270 & 12.4 \\
\hline 21 & 1.344 & NA & 1.028 & 1.463 & 1.115 & 0.820 & 58.7 \\
\hline 22 & NA & NA & 1.413 & 1.196 & 1.150 & 1.163 & 19.8 \\
\hline 23 & NA & 1.729 & NA & 1.357 & 1.402 & 1.657 & 2.2 \\
\hline 24 & NA & NA & 1.274 & NA & 1.327 & 0.971 & 9.7 \\
\hline 25 & NA & 1.385 & 1.405 & 1.466 & NA & 1.263 & 4.2 \\
\hline
\end{tabular}

Note.-NA = not applicable because MR examination was not performed at the given stroke stage. * Patients 1-16 had Tls, and patients 17-25 had deep Wls.



Figure 1. Plot illustrates mean ratios of lesion-to-tissue rCBV at MR imaging performed at six defined stages of stroke, from hyperacute $(<6$ hours) to late chronic (30-31 days). TI lesions showed a progressively increasing pattern of rCBV from hypoperfusion at hyperacute to hyperperfusion at early chronic stage. WI lesions showed persistent postischemic hyperperfusions throughout the six stages. Stages at which the mean difference between TI and WI reached statistical significance $(P<.01)$ are shown $(*)$.

pixel. Subsequently, gamma-variate fitting was applied to the concentration-time curve to remove recirculation effects. Pixel rCBV was then calculated by integrating the area under the concentration-time curve. A region of interest was drawn manually (Y.J.L.) and confirmed by a radiologist (C.Y.C.) to include the infarction region to represent the ischemic lesion. Determination of the region of interest for
rCBV analysis was based on diffusionweighted images with a $b$ of $1,000 \mathrm{sec} /$ $\mathrm{mm}^{2}$ at the acute stages because of the conspicuous contrast between lesion and normal areas. At later stages of stroke, regions of interest were encircled from diffusion-weighted images with a $b$ of $0 \mathrm{sec} /$ $\mathrm{mm}^{2}$ (intrinsically $\mathrm{T} 2$ weighted). In our experience, this procedure provides robust measurements that substantially reduce the variance of the intra- and interoperator definition of the infarct core that results from the intrinsic heterogeneity of signal intensities in ischemic lesions (7). Another same-sized region of interest homologous to the lesion was drawn to obtain the rCBV of the contralateral normal tissue. Lesionto-rCBV ratio, defined as the ratio of the mean rCBV of lesions to the mean rCBV of normal areas, was used as an indicator of possible hemodynamic abnormality. Since the main target in our study was the regulatory vasodilation following the onset of stroke symptoms, only rCBV data were reported in this study, even though other perfusion parameters, such as time to peak gadolinium concentration, were routinely used in diagnosis.

Postprocessing of multivoxel MR spectra, including phase and baseline corrections, was performed by using the software installed on the operating console (Numaris; Siemens, Erlangen, Germany). The spectral peaks corresponding to $\mathrm{N}$ acetylaspartate (NAA) and lactate were specifically examined to represent the extent of neuronal injury and oxidative metabolism, respectively. Relative concentrations of NAA were obtained by calculating the peak area of NAA after Gaussian curve fitting of the spectral peak at $2.02 \mathrm{ppm}$. For this purpose, one to three contiguous voxels in the core region of infarction were selected (Y.J.L.) according to lesion size and confirmed by a radiologist (C.Y.C.), and peak areas of NAA were recorded. NAA of normal brain tissue was also obtained from three contiguous voxels at the contralateral normal hemisphere. The lesion-to-relative NAA ratio was then determined as the mean NAA peak areas in the infarction core divided by the contralateral normal NAA. In a similar manner, the relative concentration of lactate was obtained from the same voxels in the lesion as the peak area of the spectral doublet centered at about $1.33 \mathrm{ppm}$. The lesion-to-relative lactate ratio was determined as the mean lactate peak area in the infarction core divided by the contralateral normal NAA. Note that the NAA area was used as the denominator because of the absence of lactate in the normal brain parenchyma. 


\section{Statistical Analysis}

The repeated-measures analysis of variance (ANOVA) was used to examine the differences of neuronal loss and CBV between patients with TI and those with WI, as well as among MR examinations performed at various stages of stroke. Post hoc comparisons between patients with TI and those with WI across evolutionary stages of MR examinations were performed by using the Tukey wholly significant difference tests (13), which are known to give more accurate experiment-wise type I error rates (ie, the probability that a set of multiple tests contains at least one type I error). Findings of empirical studies also showed that the wholly significant difference test is robust to the homogeneity of variance assumption and becomes slightly conservative, with unequal sample sizes having positive correlations of sample size and variance $(14,15) . P<.01$ was considered to represent a significant difference.

\section{RESULTS}

Figure 1 shows the longitudinal changes in $\mathrm{rCBV}$ measured at the six stroke stages (Table 1 also shows the infarct volume). Generally, the lesions of patients with TI had reduced rCBV in the first week after ictus, with the temporal profile of $\mathrm{rCBV}$ following a progressively increasing pattern, from lower at the hyperacute stage $(0.46 \pm 0.28)$ to higher than normal at the early chronic stage $(1.23 \pm 0.34)$. The rCBV value then decreased, but not significantly, to $0.86 \pm 0.26$ at 1 month following stroke symptom onset. In patients with WI, there was persistent postischemic increase in $\mathrm{rCBV}$ throughout the six stages $(1.34,1.33 \pm 0.42,1.11 \pm$ $0.27,1.25 \pm 0.19,1.23 \pm 0.30$, and $1.18 \pm 0.32$ for hyperacute, acute, early acute, late subacute, early chronic, and late chronic, respectively). Repeated-measures ANOVA showed that there was a significant difference $(P<.004)$ between the two subtypes of infarction, thus indicating that the evolutionary patterns associated with rCBV across the stages between TI and WI were significantly different. At the individual stage, however, the wholly significant difference test findings suggested that rCBV values differed between the two subtypes at the very beginning.

Figure 2 shows the longitudinal changes of relative NAA from hyperacute to late chronic stages of ischemic stroke, with individual data listed in Table 2. Patients with both TI and WI showed initial decrease of relative NAA at the hy-

\begin{tabular}{|c|c|c|c|c|c|c|}
\hline $\begin{array}{l}\text { Patient } \\
\text { No.* }\end{array}$ & Hyperacute & Acute & $\begin{array}{c}\text { Early } \\
\text { Subacute }\end{array}$ & $\begin{array}{c}\text { Late } \\
\text { Subacute }\end{array}$ & $\begin{array}{c}\text { Early } \\
\text { Chronic }\end{array}$ & $\begin{array}{l}\text { Late } \\
\text { Chronic }\end{array}$ \\
\hline 1 & NA & 0.186 & NA & 0.151 & 0.212 & 0.147 \\
\hline 2 & NA & NA & 0.051 & 0.276 & 0.050 & NA \\
\hline 3 & NA & 0.356 & NA & 0.163 & 0.101 & 0.149 \\
\hline 4 & NA & NA & 0.286 & NA & 0.367 & 0.063 \\
\hline 5 & 0.987 & NA & 0.320 & 0.289 & 0.117 & 0.203 \\
\hline 6 & 0.459 & NA & 0.191 & NA & NA & 0.173 \\
\hline 7 & 0.479 & NA & 0.167 & 0.174 & NA & 0.033 \\
\hline 8 & NA & NA & 0.149 & 0.206 & 0.366 & 0.323 \\
\hline 9 & NA & NA & 0.193 & 0.218 & 0.337 & 0.342 \\
\hline 10 & NA & NA & 0.347 & 0.145 & 0.097 & 0.246 \\
\hline 11 & NA & 0.651 & NA & 0.404 & NA & NA \\
\hline 12 & NA & NA & 0.402 & 0.354 & 0.409 & NA \\
\hline 13 & 0.704 & NA & NA & 0.411 & 0.390 & NA \\
\hline 14 & NA & NA & 0.410 & NA & 0.415 & 0.428 \\
\hline 15 & NA & NA & 0.499 & 0.383 & 0.256 & 0.210 \\
\hline 16 & NA & 0.630 & 0.522 & 0.519 & NA & 0.387 \\
\hline 17 & NA & NA & 0.452 & 0.460 & 0.514 & 0.269 \\
\hline 18 & NA & 0.903 & NA & 0.908 & 0.811 & NA \\
\hline 19 & NA & NA & 0.668 & NA & 0.603 & 0.684 \\
\hline 20 & NA & NA & 0.686 & 0.500 & 0.330 & 0.325 \\
\hline 21 & 0.800 & NA & 0.616 & 0.450 & 0.662 & 0.575 \\
\hline 22 & NA & NA & 0.443 & 0.486 & 0.205 & 0.153 \\
\hline 23 & NA & 0.847 & NA & 0.925 & 0.797 & 0.905 \\
\hline 24 & NA & NA & 0.450 & NA & 0.554 & 0.591 \\
\hline 25 & NA & 0.883 & 0.674 & 0.784 & NA & 0.700 \\
\hline
\end{tabular}

Note.-NA = not applicable because MR examination was not performed at the given stroke stage. * Patients 1-16 had Tls, and patients 17-25 had deep Wls.

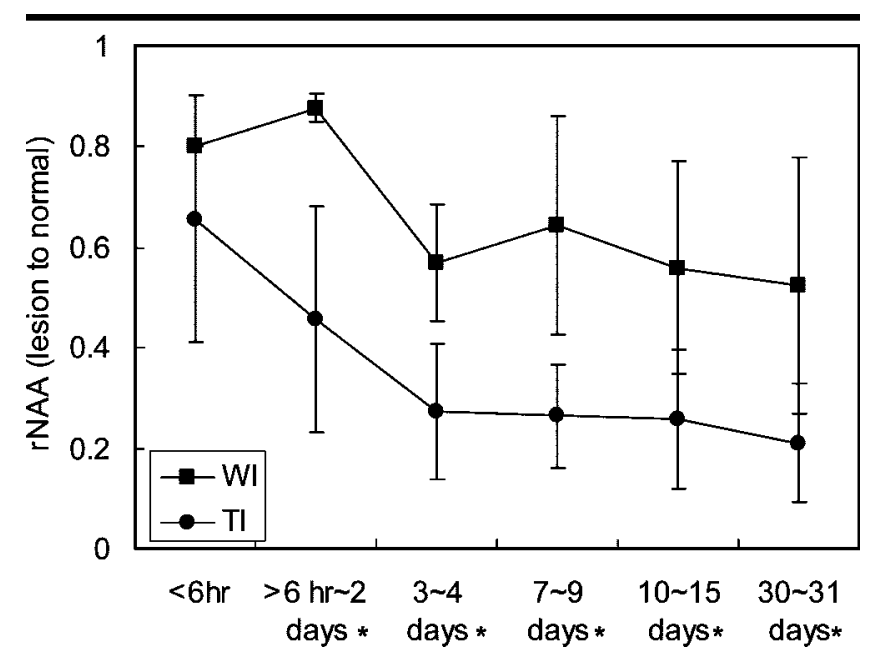

Figure 2. Plot illustrates mean relative NAA values at six defined stages of TI and WI, from hyperacute ( $<6$ hours) to late chronic (30-31 days). Patients with TI showed complete loss of relative NAA within 4 days after ictus. Patients with WI showed higher relative NAA ( 0.8 at acute stage and about 0.6 at the four subsequent stages), implying neuron survival. Stages at which the mean difference between TI and WI reached statistical significance $(P<.01)$ are shown $(*)$.

peracute stage. The relative NAA of patients with TI decreased to about one-half $(0.46 \pm 0.22)$ within 2 days after onset of stroke symptoms and was lost completely within 4 days after ictus. Note that the relative NAA values for patients with TI were all less than 0.3 from the early subacute to the late chronic stage, which were close to the level of noise. In contrast, patients with WI showed higher relative NAA values than did patients with $\mathrm{TI}$, as represented by values greater than 
TABLE 3

Relative Lactate Values Obtained at Various Stroke Stages

\begin{tabular}{|c|c|c|c|c|c|c|}
\hline $\begin{array}{c}\text { Patient } \\
\text { No.* }\end{array}$ & Hyperacute & Acute & $\begin{array}{c}\text { Early } \\
\text { Subacute }\end{array}$ & $\begin{array}{c}\text { Late } \\
\text { Subacute }\end{array}$ & $\begin{array}{c}\text { Early } \\
\text { Chronic }\end{array}$ & $\begin{array}{c}\text { Late } \\
\text { Chronic }\end{array}$ \\
\hline 1 & NA & 2.572 & NA & 0.464 & 0.584 & 0.400 \\
\hline 2 & NA & NA & 3.135 & 0.540 & 0.537 & NA \\
\hline 3 & NA & 3.797 & NA & 1.765 & 0.846 & 0.701 \\
\hline 4 & NA & NA & 0.730 & NA & 0.570 & 0.436 \\
\hline 5 & 1.018 & NA & 0.305 & 0.590 & 0.390 & 0.548 \\
\hline 6 & 2.431 & NA & 1.864 & NA & NA & 0.293 \\
\hline 7 & 0.941 & NA & 1.141 & 1.440 & NA & 1.973 \\
\hline 8 & NA & NA & 0.585 & 0.114 & 0.137 & 0.195 \\
\hline 9 & NA & NA & 0.433 & 0.539 & 0.108 & 2.212 \\
\hline 10 & NA & NA & 1.020 & 1.144 & 0.516 & 0.328 \\
\hline 11 & NA & 1.632 & NA & 1.021 & NA & NA \\
\hline 12 & NA & $\mathrm{NA}$ & 0.645 & 0.668 & 0.401 & NA \\
\hline 13 & 1.368 & NA & NA & 1.010 & 0.436 & NA \\
\hline 14 & NA & NA & 0.748 & NA & 0.544 & 0.335 \\
\hline 15 & NA & NA & 0.960 & 0.652 & 0.144 & 0.187 \\
\hline 16 & NA & 0.838 & 0.857 & 1.025 & NA & 0.562 \\
\hline 17 & NA & NA & 0.822 & 0.632 & 0.887 & 0.374 \\
\hline 18 & NA & 0.527 & NA & 0.310 & 0.192 & NA \\
\hline 19 & NA & NA & 0.500 & NA & 0.034 & 0.301 \\
\hline 20 & NA & NA & 0.643 & 0.842 & 0.494 & 0.324 \\
\hline 21 & 0.358 & NA & 0.115 & 0.342 & 0.100 & 0.322 \\
\hline 22 & NA & NA & 0.844 & 0.953 & 0.788 & 0.629 \\
\hline 23 & NA & 0.963 & NA & 0.445 & 0.416 & 0.304 \\
\hline 24 & NA & NA & 0.538 & NA & 0.119 & 0.240 \\
\hline 25 & NA & 0.285 & 0.331 & 0.406 & NA & 0.468 \\
\hline
\end{tabular}

Note.-NA = not applicable because MR examination was not performed at the given stroke stage. * Patients 1-16 had Tls, and patients 17-25 had deep Wls.

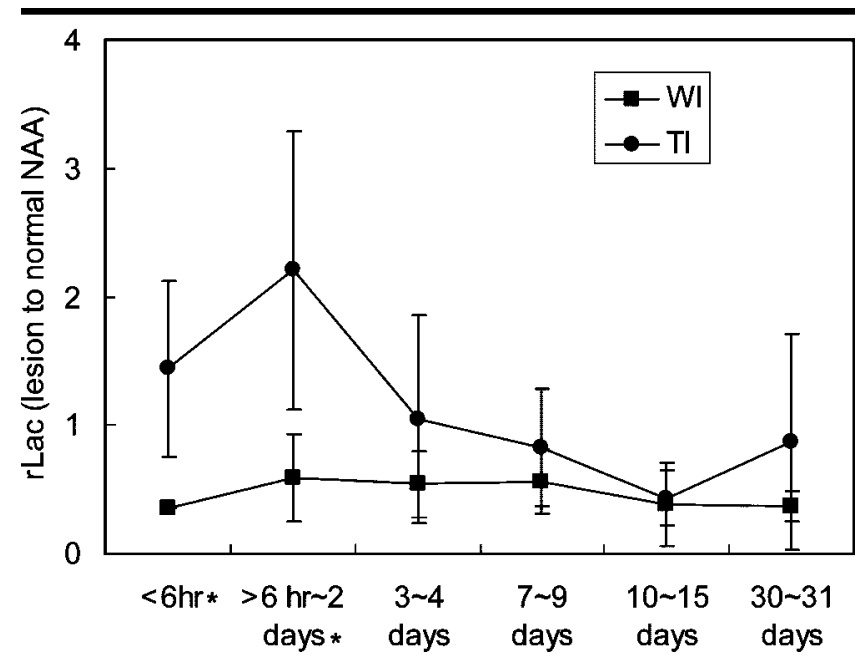

Figure 3. Plot illustrates mean relative lactate values at six defined stages of TI and WI, from hyperacute ( $<6$ hours) to late chronic (30-31 days). Patients with TI exhibited high levels of lactate at hyperacute $(1.44 \pm 0.69)$ and acute $(2.21 \pm 1.27)$ stages. Patients with WI showed relatively stable relative lactate values between about 0.4 and 0.6. Stages at which the mean difference between TI and WI reached statistical significance $(P<.01)$ are shown $(*)$.

$0.8(0.88 \pm 0.03)$ in the acute stage and about 0.6 for the four subsequent stages $(0.57 \pm 0.12,0.64 \pm 0.22,0.56 \pm 0.21$, and $0.53 \pm 0.25$ at early subacute, late subacute, early chronic, and late chronic, respectively). Repeated-measures ANOVA showed that the longitudinal evolution of relative NAA between TI and WI was significantly different $(P<.0001)$. The findings of post hoc comparison with Tukey wholly significant difference tests showed that relative NAA was significantly differ- ent for the two groups of patients at all stages but the hyperacute stage $(P<.01)$.

Similar to Figure 2, Figure 3 shows the longitudinal changes of relative lactate measured at the six stages for both groups of patients (Table 3 ). Patients with TI exhibited high levels of lactate at the hyperacute stage $(1.44 \pm 0.69)$, and the levels were particularly prominent at the acute stage $(2.21 \pm 1.27)$. At the four subsequent stages, the peak areas of lactate doublets decreased to about the level of normal NAA or even lower (1.04 \pm $0.77,0.84 \pm 0.45,0.43 \pm 0.22,0.87 \pm$ 0.84 for early subacute, late subacute, early chronic, and late chronic stages, respectively). Patients with WI showed relatively stable relative lactate values between about 0.4 and $0.6(0.36,0.59 \pm$ $0.34,0.54 \pm 0.26,0.56 \pm 0.25,0.38 \pm$ 0.33 , and $0.37 \pm 0.12$ for hyperacute, acute, early subacute, late subacute, early chronic, and late chronic stages, respectively). Repeated-measures ANOVA showed that the longitudinal evolution of relative lactate between TI and WI was significantly different $(P<.01)$. Findings of post hoc comparison showed that relative lactate values were significantly lower in WI than those in TI at the acute stage $(P<.01)$. Note that although differences in hyperacute relative lactate values between WI and TI also reached statistical significance $(P<.01)$, the significance is weak owing to small sample size $(n=1$ for $\mathrm{WI})$.

Figures 4 and 5 show one typical case each from the TI and WI, respectively, groups. Differences in the evolution patterns for relative ADC (7), rCBV, relative NAA, and relative lactate are clearly depicted. Table 4 shows the $P$ values and 99\% CIs for the post hoc comparison with Tukey wholly significant difference tests that detail the statistical difference between the two infarction subtypes (TI compared with WI).

\section{DISCUSSION}

The temporal changes of deep WI versus TI are different in many aspects. A recent investigation about ADC evolution in human ischemic stroke shows that patients with WI demonstrated a slower pseudonormalization (about a month after ictus) than did patients with TI (around 10 days after onset) following the initial acute decline of ADC (7). This subtype dependence on the speed of infarction progression is anticipated to be associated with hemodynamics, because findings of earlier animal studies have 


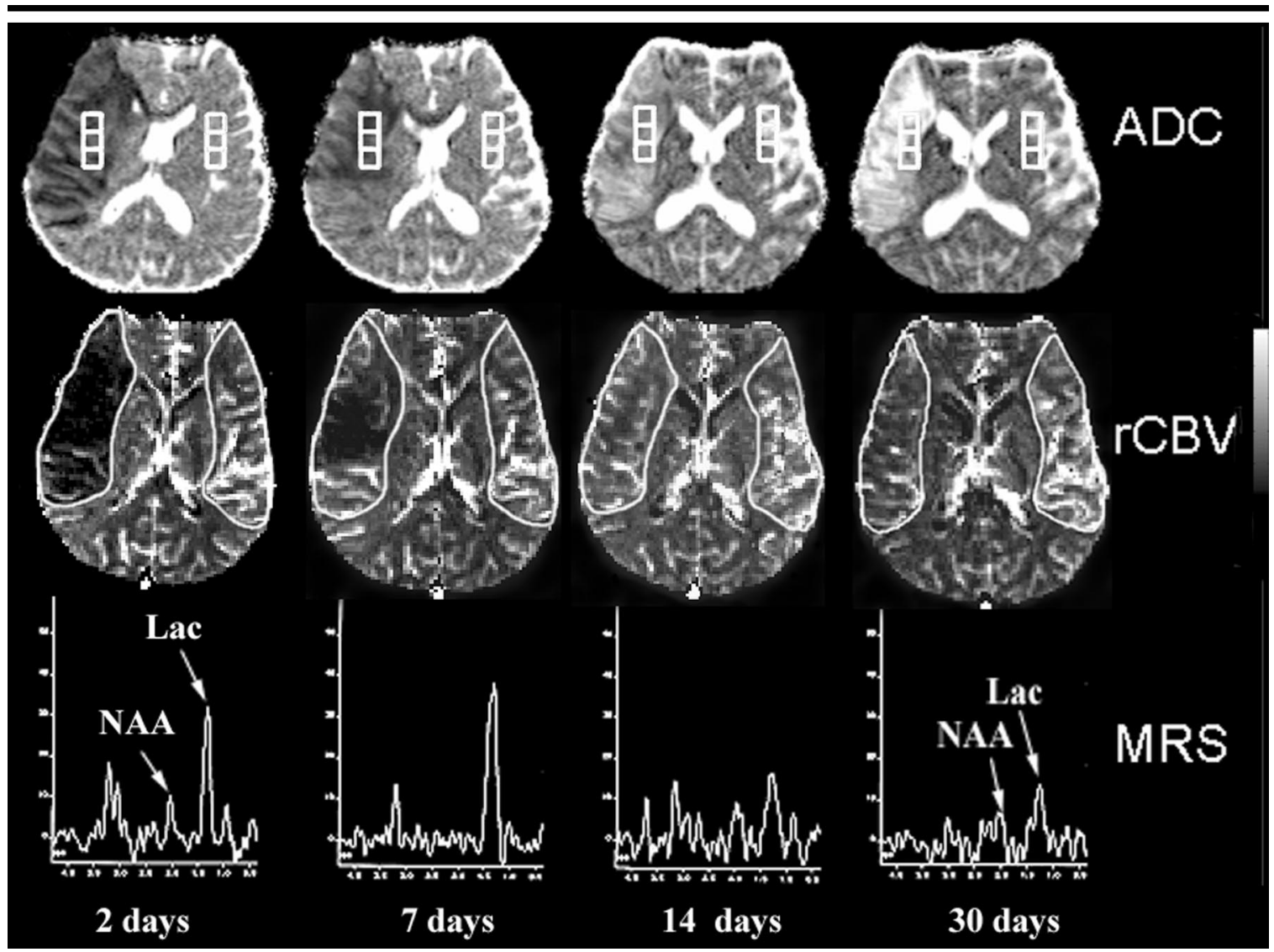

Figure 4. Time course of TI of the right middle cerebral artery, as demonstrated on ADC maps (top row), rCBV maps (middle row), and proton MR spectra (bottom row) at 2 (first image from left), 7 (second image from left), 14 (third image from left), and 30 (fourth image from left) days after stroke symptom onset in a 51-year-old male patient. Rectangles on the ADC maps indicate the voxels included for spectral analysis, and white borders show the region of interest for rCBV analysis. Pseudonormalization of ADC commenced at about 2 weeks after ictus, before which the evolution of postischemic reperfusion can be clearly depicted on the rCBV maps. Both loss of relative NAA and significant presence of relative lactate at acute stage are seen in the spectra.

shown temporal alterations in ADC to be closely related to postischemic tissue perfusion $(9,10)$.

Our longitudinal investigation about both the hemodynamics and the metabolic status in which human subjects with natural stroke progression were studied provides a consolidating evidence for the subtype-dependent temporal evolutions as stated previously. In addition to ADC alterations demonstrated previously (7), the results of our study further indicate that CBV and neuronal injury are also evolutionally dependent on infarction subtypes. In particular, the neuronal injury, as represented by changes in relative NAA, is slower in WI than in TI, which is similar to and consistent with the behavior of ADC evolution (7).

The temporal profiles of $\mathrm{rCBV}$ for the two distinct subtypes of infarctions revealed important messages regarding the mechanisms of stroke evolution. For pa- tients with TI, rCBV follows a progressively increasing pattern, from lower values at the hyperacute stage to higher than normal values at the early chronic stage. The reduced rCBV in the hyperacute stage is obviously the consequence of a severe vascular compromise from the abrupt artery occlusion. Reperfusion starts after the acute stage, with maximum rCBV higher than that of the normal brain tissue, which is reached at about the late subacute stage. The early reperfusion is accompanied by abnormal vasodilatation (increased rCBV) and decreased oxygen extraction fraction (nonnutritional "luxury perfusion"), which was suggested as postischemic rebound of cellular metabolism in structurally preserved tissue $(16,17)$.

In contrast to $\mathrm{TI}$, the temporal profile of rCBV in patients with WI shows a sustained high value in the ischemic tissue throughout the six stages investigated in this study. Since WI has classically been thought to arise from a critical reduction in cerebral blood supply (ie, a hemodynamic mechanism) due to previous existence of vessel stenosis $(1,8)$, an increase in $\mathrm{rCBV}$ is anticipated to reflect autoregulatory vasodilation in order to reduce vascular resistance in response to decreased cerebral perfusion pressure (18). Furthermore, the relatively stable rCBV values seen in patients with TI suggested that maximum compensatory vasodilation may have already existed in the ischemic tissues at the early stages of symptom onset. This preexisting vasodilation could additionally reduce the vascular response to extrinsic stimuli such as acidosis (19), resulting in the absence of significant temporal changes in $\mathrm{rCBV}$.

The distinct temporal changes in brain metabolites in TI and WI may also imply different pathogenetic mechanisms between these two infarction subtypes. The lower level of relative lactate indicates that oxidative metabolism might have 
TABLE 4

Post Hoc Comparison Results of Tukey Wholly Significant Difference Test for TI versus WI

\begin{tabular}{lcccccc}
\hline Parameter & Hyperacute & Acute & Early Subacute & Late Subacute & Early Chronic & Late Chronic \\
\hline rCBV & $<.00004(-1.4,-.35)$ & $<.001(-1.2,-.15)$ & $>.05(\mathrm{NA})$ & $>.05(\mathrm{NA})$ & $>.05(\mathrm{NA})$ & $>.05(\mathrm{NA})$ \\
Relative & $>.05(\mathrm{NA})$ & $<.00005(-.67,-.17)$ & $<.005(-.52,-.03)$ & $<.0003(-.61,-.11)$ & $<.002(-.55,-.05)$ & $<.002(-.55,-.05)$ \\
$\quad$ NAA & $<.002(.20,2.0)$ & $<.00001(.73,2.5)$ & $>.05(\mathrm{NA})$ & $>.05(\mathrm{NA})$ & $>.05(\mathrm{NA})$ & $>.05(\mathrm{NA})$ \\
$\begin{array}{r}\text { Relative } \\
\quad \text { lactate }\end{array}$ & $<.07$
\end{tabular}

Note.-Data are $P$ values. Data in parentheses are $99 \%$ Cls. NA = not applicable because statistical significance was not reached.

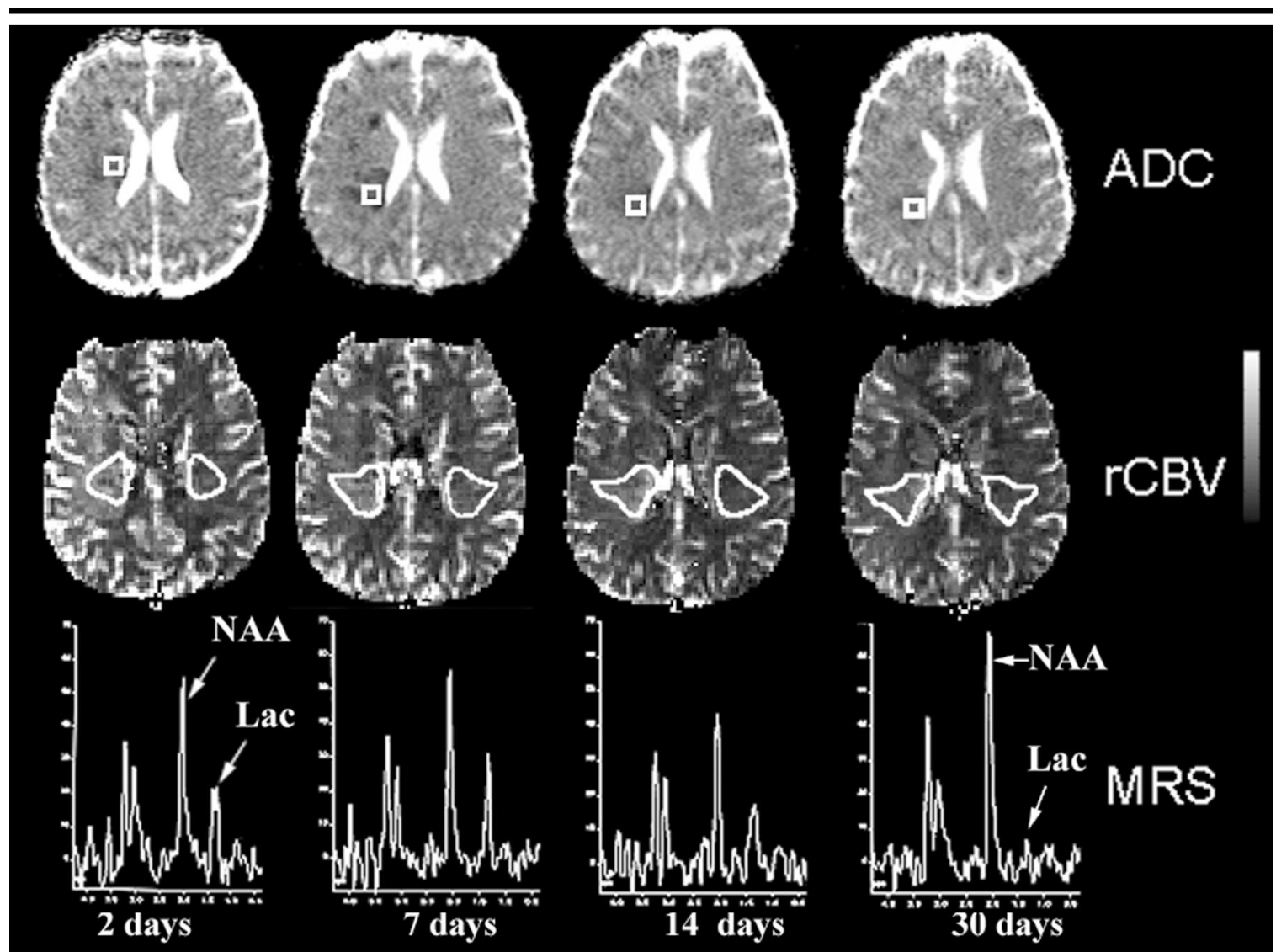

Figure 5. Time course of WI of the right middle cerebral artery, as depicted on ADC maps (top row), rCBV maps (middle row), and proton MR spectra (bottom row) at 2 (first image from left), 7 (second image from left), 14 (third image from left), and 30 (fourth image from left) days after stroke symptom onset in a 57-year-old female patient. Rectangles on the ADC maps indicate the voxels included for spectral analysis, and white borders show the region of interest for rCBV analysis. ADC evolution is much less significant. Persistently increased rCBV is seen. Relative NAA decreased only partially (compared with normal tissue, spectra not shown), with relative lactate level varying from 0.9 (acute) to 0.3 (late chronic).

failed only partially in patients with WI at the acute stage. This would suggest unexhausted oxygen supplied by increased oxygen extraction along with an increased rCBV that maintains oxidative metabolism and brain function when cerebral perfusion pressure is reduced (20). Patients with TI, on the other hand, exhibit a substantially higher relative lactate level than do those with WI during the acute stage, which reflects anaerobic glycolysis as the main source of energy metabolism. This metabolic difference might account in part for the differing ADC evolutions between TI and WI reported in a previous article (7). The decrease of relative lactate in patients with TI at subsequent stages may be attributed to increased perfusion, which facilitates lactate removal from the damaged tissue into the bloodstream through passive diffusion in the presence of blood-brain bar- rier disruption $(21,22)$. In other words, evolution of relative lactate for the two subtypes of infarction possibly reflects a consequence of different underlying hemodynamics. Alternatively, the reduced demand of cerebral metabolism with complete loss of living neurons at about 4 days after symptom onset could be another reason for the reduced relative lactate level in patients with TI.

The progress of neuronal injury repre- 


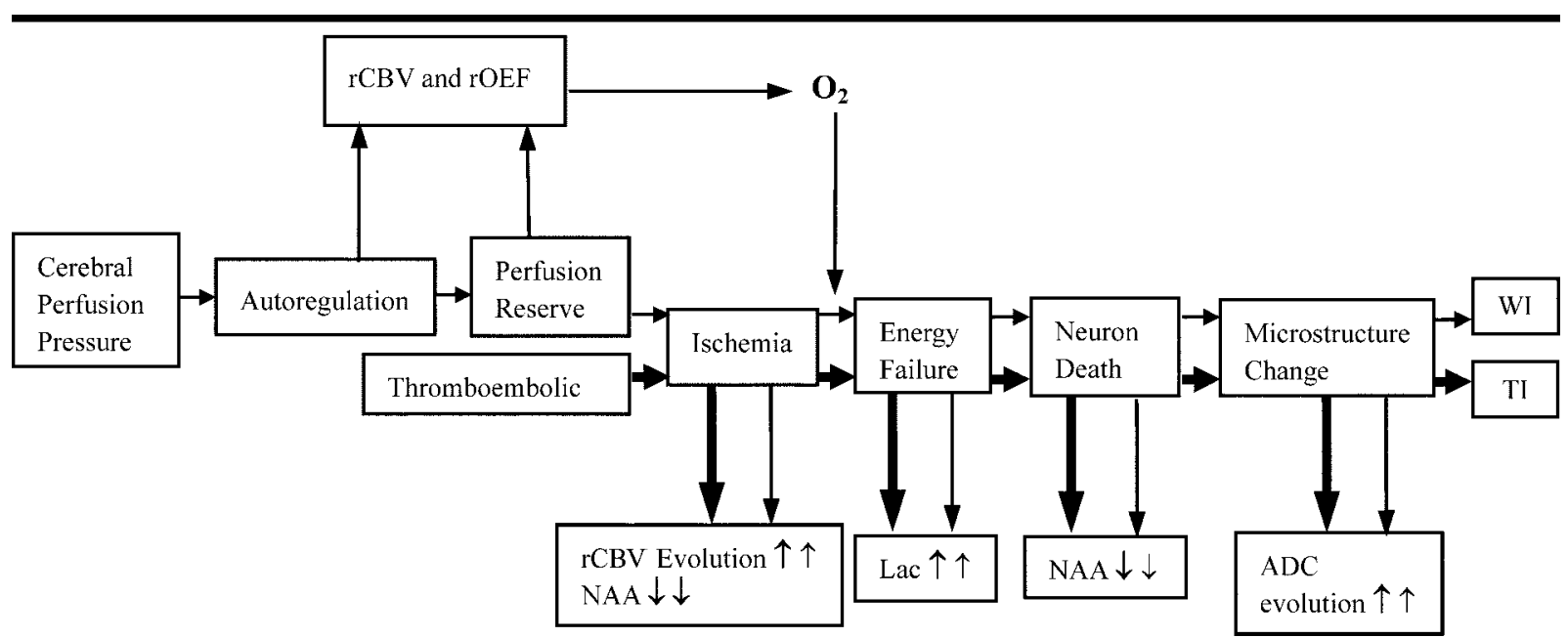

Figure 6. Proposed model for pathophysiologic time course of human ischemic stroke. Thicker arrows indicate more significant and faster changes. WI likely results from a preexisting reduction in cerebral perfusion pressure that induces cerebral autoregulation with rCBV increase. Possible elevated oxygen extraction fraction further protects the neurons from experiencing energy deficiency. These factors lead to a generally slower stroke progression. On the other hand, TI causes an abrupt cerebral ischemia, without a chance to develop sufficient collateral arteries during the critical period. As a result, complete loss of living neurons occurs early, and subsequent alterations in $\mathrm{rCBV}$ are deemed ineffective. $L a c=$ lactate, $r O E F=$ relative oxygen extraction fraction.

sented by a decline of relative NAA is slower in WI than in TI, which is consistent with previous findings regarding infarction subtype-dependent ADC evolution (7). Results of our study indicate complete neuronal loss in TI at about 4 days following the onset of symptoms. The significant presence of inefficient anaerobic glycolysis in TI during acute stages is possibly the major origin for an eventual energy failure, which leads to the complete loss of living neurons (23$25)$. In patients with WI, however, residual relative NAA of $0.53 \pm 0.25$ was found during the late chronic stage, which suggests neuron survival even after 1 month after ictus. With preexisting vascular autoregulation plus the elevated oxygen extraction fraction as mentioned earlier for WI, neuron survival is more likely to result (26). In addition, a clinical investigation regarding hyperacute ischemic stroke suggests that an increased rCBV likely corresponds to preexisting compensatory vasodilation caused by collateral circulation, which leads to generally better clinical outcomes (5). The slower relative NAA decline depicted for WI is in good agreement with this finding, further supporting the hypothesis that the subtype-dependent progression of neuronal injury is associated with the pathophysiologic hemodynamics.

An integration of the aforementioned information obtained in our study has allowed us to construct a model for the pathophysiologic time course of human ischemic stroke. As demonstrated in Fig- ure 6, WI is likely to arise from a preexisting reduction in cerebral perfusion pressure due to arterial stenosis. The cerebral autoregulation fulfills metabolic demands of the living brain tissues with an increased rCBV through compensatory vasodilation (ie, an opening of the perfusion reserve). Possible elevated oxygen extraction fraction further protects the neurons from experiencing energy deficiency. Overall, the factors lead to a generally slower temporal stroke progression following the critical hypoperfusion that causes cerebral ischemia. On the other hand, the thromboembolic nature of TI causes abrupt cerebral ischemia without a chance to develop sufficient collateral arteries during the critical period. Subsequent alterations in rCBV are deemed ineffective. As a result, complete loss of living neurons occurs at as early as 4 days after ischemic attack. Our observations may implicate that the previous ineffective trials regarding the treatment of ischemic stroke with neuroprotective agents can be reevaluated in the WI patient group, considering the long evolution of stroke (27).

One potential weakness of our study is the lack of a large patient population, especially patients with hyperacute stroke data $(n=4$ for TI and $n=1$ for WI). Therefore, temporal progression of pathophysiologic factors for ischemic stroke during the acute stage in TI and WI cannot be firmly established from our data. Hence, we regard our study as providing strong evidence, rather than firm proof, of the hemodynamic and neuronal injury evolutions in TI and WI. Clearly, further studies at a larger scale are desirable. At our institute, however, the availability of aggressive interventional treatments such as intraarterial thrombolytic therapy plus our increasing experience in acute stroke image interpretations has ethically precluded us from collecting a substantially larger number of cases with natural stroke progression for the follow-through study.

Partial volume effects could be a potential source of error for the spectroscopic results, especially considering that WI lesions are often smaller than TI lesions. In this study, partial volume effects were minimized with careful control of the anatomic location from which the spectra were acquired. Since all the infarct volumes reported in this study were larger than the voxel size (acquisition volume, $10 \times 10 \times 10 \mathrm{~mm}^{3}$ ) at chemical shift imaging, and zero filling was applied to further reduce the chemical shift imaging voxel volume to $5 \times 5 \times 10 \mathrm{~mm}^{3}$ for analysis, biasing of the results is anticipated to be minimal.

In conclusion, findings of our longitudinal study show clearly distinct temporal patterns in both the hemodynamics and the metabolism for two types of infarctions: TI and WI. We believe that the most important factor determining the speed of progression lies in the pathophysiologic hemodynamics, an understanding of which may have important implications in future patient treatments. 


\section{References}

1. Ringelstein EB, Zeumer H, Angelou D. The pathogenesis of strokes from internal carotid artery occlusion: diagnostic and therapeutical implications. Stroke 1983; 14:867-875.

2. Moriwaki H, Matsumoto M, Hashikawa K, et al. Hemodynamic aspect of cerebral watershed infarction: assessment of perfusion reserve using iodine-123-iodoamphetamine SPECT. J Nucl Med 1997; 38: $1556-1562$.

3. Bogousslavsky J, Regli F. Unilateral watershed cerebral infarcts. Neurology 1986; 36:373-377.

4. Weiller C, Ringelstein EB, Reiche W, Buell U. Clinical and hemodynamic aspects of low-flow infarcts. Stroke 1991; 22:11171123.

5. Kim JH, Shin T, Park JH, Chung SH, Choi $\mathrm{NC}$, Lim BH. Various patterns of perfusion-weighted MR imaging and MR angiographic findings in hyperacute ischemic stroke. AJNR Am J Neuroradiol 1999; 20:613-620.

6. Caplan LR, Mohr PJ, Kistler JP, Koroshetz W. Should thrombolytic therapy be the first-line treatment for acute ischemic stroke? N Engl J Med 1997; 337:13091310 .

7. Huang IJ, Chen CY, Chung HW, et al. Time course of cerebral infarction in the middle cerebral arterial territory: deep watershed versus territorial subtypes on diffusion-weighted MR images. Radiology 2001; 221:35-42.

8. Bladin CF, Chambers B. Frequency and pathogenesis of hemodynamic stroke. Stroke 1994; 25:2179-2182.

9. Rother J, de Crespigny AJ, D'Arceuil H, Iwai K, Moseley ME. Recovery of apparent diffusion coefficient after ischemia-induced spreading depression relates to cerebral perfusion gradient. Stroke 1996; 27:980-987.
10. Dijkhuizen RM, Berkelbach van der Sprenkel JW, Tulleken KA, Nicolay K. Regional assessment of tissue oxygenation and the temporal evolution of hemodynamic parameters and water diffusion during acute focal ischemia in rat brain. Brain Res 1997; 750:161-170.

11. Damasio H. A computed tomographic guide to the identification of cerebral vascular territories. Arch Neurol 1983; 40: 138-142.

12. Belliveau JW, Rosen BR, Kantor HL, et al. Functional cerebral imaging by susceptibility-contrast NMR. Magn Reson Med 1990; 14:538-546.

13. Games PA. Alternative analyses of repeatedmeasure designs by ANOVA and MANOVA In: Alexander VE, ed. Statistical methods in longitudinal research. Vol 1, Principles and structuring change. New York, NY: Academic Press, 1990.

14. Keselman HJ. A power investigation of the Tukey multiple comparison statistics. Educ Psychol Meas 1975; 36:91-104.

15. Keselman HJ, Toothaker LE. Comparison of Tukey's T-method and Scheffe's S-method for various numbers of all possible differences of averages contrasts under violations of assumptions. Educ Psychol Meas 1974; 34:511-520.

16. Baron JC, Frackowiak RSJ, Herholz K, et al. Use of PET methods for measurement of cerebral energy metabolism and hemodynamics in cerebrovascular disease. J Cereb Blood Flow Metab 1989; 9:723742.

17. Marchal G, Young AR, Baron JC. Early postischemic hyperperfusion: pathophysiologic insights from positron emission tomography. J Cereb Blood Flow Metab 1999; 19:467-482.

18. Furst H, Hartl WH, Janssen I. Patterns of cerebrovascular reactivity in patients with unilateral asymptomatic carotid artery stenosis. Stroke 1994; 25:1193-1200.

19. McKinnon W, Aaronson PI, Knock G,
Graves J, Poston L. Mechanism of lactateinduced relaxation of isolated rat mesenteric resistance arteries. J Physiol 1996; 490:783-792.

20. Powers WJ. Cerebral hemodynamics in ischemic cerebrovascular disease. Ann Neurol 1991; 29:231-240.

21. Graham GD, Blamire AM, Rothman DL, et al. Early temporal variation of cerebral metabolites after human stroke: a proton magnetic resonance spectroscopy study. Stroke 1993; 24:1891-1896.

22. Saunders DE. MR spectroscopy in stroke. Br Med Bull 2000; 56:334-345.

23. Bruhn H, Frahm J, Gyngell ML, Merboldt KD, Hanicke W, Sauter R. Cerebral metabolism in man after acute stroke: new observations using localized proton NMR spectroscopy. Magn Reson Med 1989; 9:126-131.

24. Rosenberg GA, White J, Gasparovic C, Crisostomo EA, Griffey RH. Effect of hypoxia on cerebral metabolites measured by proton nuclear magnetic resonance spectroscopy in rats. Stroke 1991; 22:73-79.

25. Barker PB, Gillard JH, van Zijl PC, et al. Acute stroke: evaluation with serial proton MR spectroscopic imaging. Radiology 1994; 192:723-732.

26. Young AR, Sette G, Touzani O, et al. Relationships between high oxygen extraction fraction in the acute stage and final infarction in reversible middle cerebral artery occlusion: an investigation in anesthetized baboons with positron emission tomography. J Cereb Blood Flow Metab 1996; 16:1176-1188.

27. Morgenstern LB. What have we learned from clinical neuroprotective trials? Neurology 2001; 57:S45-S47. 